\title{
SUBMILLIMETER-WAVE ANTENNAS ON THIN MEMBRANES
}

\author{
Gabriel M. Rebeiz, Wade G. Regehr and David B. Rutledge \\ Division of Engineering and Applied Science \\ California Institute of Technology \\ Pasadena, CA 91125 \\ Richard L. Savage, and Neville C. Luhmann Jr. \\ Department of Electrical Sciences \\ University of California, Los Angeles \\ Los Angeles, CA 90024
}

\begin{abstract}
Submillimeter-wave antennas with bismuth microbolometer detectors have been fabricated on 1- $\mu \mathrm{m}$ thick silicon-oxynitride membranes. This approach results in better patterns than previous lens-coupled integrated circuit antennas, and eliminates the dielectric loss associated with the substrate lens. Measurements on a wideband log-periodic antenna at $700 \mathrm{GHz}, 380 \mathrm{GHz}$ and $167 \mathrm{GHz}$, and on a $700 \mathrm{GHz}$ logperiodic imaging array, show no sidelobes and a $3-\mathrm{dB}$ beamwidth between $40^{\circ}$ and $50^{\circ}$. Also, the effective area can be increased by $5 \mathrm{~dB}$ by the use of a back-shorting mirror. Possible application areas are superconducting tunnel junction receivers for radio astronomy and imaging arrays for plasma diagnostics.

Introduction

At millimeter and submillimeter wavelengths, integrated circuit antennas are often mounted on a substrate lens to eliminate losses due to substrate modes $[1,2]$. This takes advantage of the fact that antennas on dielectrics are more sensitive to radiation from the substrate. However typical coupling efficiencies for these antennas have been limited to around $25 \%$ because of poor antenna patterns and dielectric losses. The dielectric losses become particularly severe at submillimeter wavelengths. We have solved both problems by fabricating the antennas on thin membranes (Fig. 1). The membrane is very thin compared to a free space wavelength, so that the antenna effectively radiates in free space. This greatly reduces the dielectric losses, and allows the use of free-space antenna designs and techniques. A back-plane reflector is needed to make the pattern unidirectional.
\end{abstract}

\section{Fabrication}

A $1-\mu \mathrm{m}$ silicon-oxynitride layer is deposited on both sides of a $<100>$ silicon wafer using plasma-enhanced chemical vapor deposition. The layer must be in tension to yield flat and rigid self-supporting membranes. These are fabricated in two steps. First, an opening is defined on the back of the wafer by patterning the silicon oxynitride with photoresist and etching it in a bufferedHF solution. Then the silicon is etched in an ethylenediamine-pyrocatechol CH2435-6/87/0000-1194\$01.00 1987 IEEE 


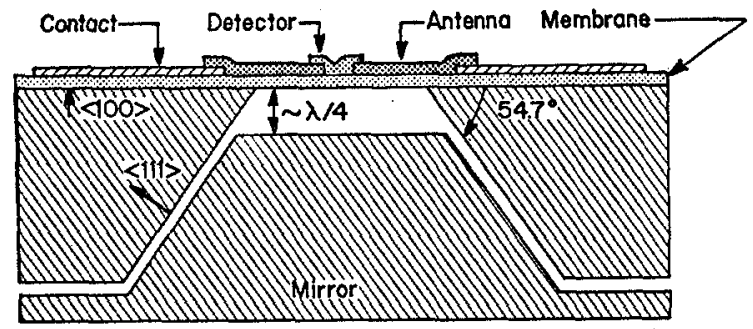

Figure 1. Antenna on thin membrane with mirror in etched trough.

solution [3] until the transparent membrane appeared. The etching process is anisotropic and forms pyramidal cavities bounded $<111>$ crystal planes. These side walls form an angle of $54.7^{\circ}$ with the $<100>$ wafer plane. Next the antennas and detectors are lithographically defined on the membrane. The detectors are bismuth microbolometers with a DC resistance of about $150 \Omega$. The DC responsivity of the detectors on the membrane was about $10 \mathrm{~V} / \mathrm{W}$ at a bias of $0.1 \mathrm{~V}$, comparable to similar detectors on quartz [4].

The mirror fabrication follows the same process. An oxide layer is thermally grown on both sides of a $<100>$ silicon wafer. The oxide is stripped off on one side except for patches which are slightly larger than the membrane. Then the silicon wafer is etched down to the desired depth. This patterning produces mesas bounded by the $\langle 111\rangle$ planes. However the patch corners are not protected and considerable lateral etching occurs. This results in a roughly circular mesa. Finally a layer of gold is evaporated on the mirror structure to yield a highly reflective surface.

\section{Wideband log-periodic antenna}

The antenna follows the classical design of Duhamel and Isbell $[5,6]$ with $\tau=0.5$ and $\sigma=\sqrt{\tau}=0.707$ (Fig. 2). The antenna is a self-complementary design with an impedance of $190 \Omega$ over the frequency range from $100 \mathrm{GHz}$ to $1400 \mathrm{GHz}$. The measurements were done at $700 \mathrm{GHz}, 380 \mathrm{GHz}$ and $167 \mathrm{GHz}$ (Fig. 2,3). The antenna was always linearly polarized along the resonant tooth, with a measured cross polarization ratio of $20 \mathrm{~dB}$. The structure in the $167 \mathrm{GHz}$ pattern is due to the interaction of the resonant tooth lying near the edge of the membrane with the neighboring silicon.

Measurements with a back-shorting mirror were done at $380 \mathrm{GHz}$ and $167 \mathrm{GHz}$ (Fig. 4). The mirror dimension was around $1.75 \mathrm{~mm}$ to fit a $1.85 \mathrm{~mm}$ membrane, and was aligned with a He-Ne laser. A 3-dB increase in the effective area was achieved at $\lambda / 4$ and a $5-\mathrm{dB}$ increase at $3 \lambda / 4$. The measured patterns at $\lambda / 4$ were very similar to the patterns without a mirror, and the polarization properties of the antenna were not changed. No pattern measurements were done at $3 \lambda / 4$. 

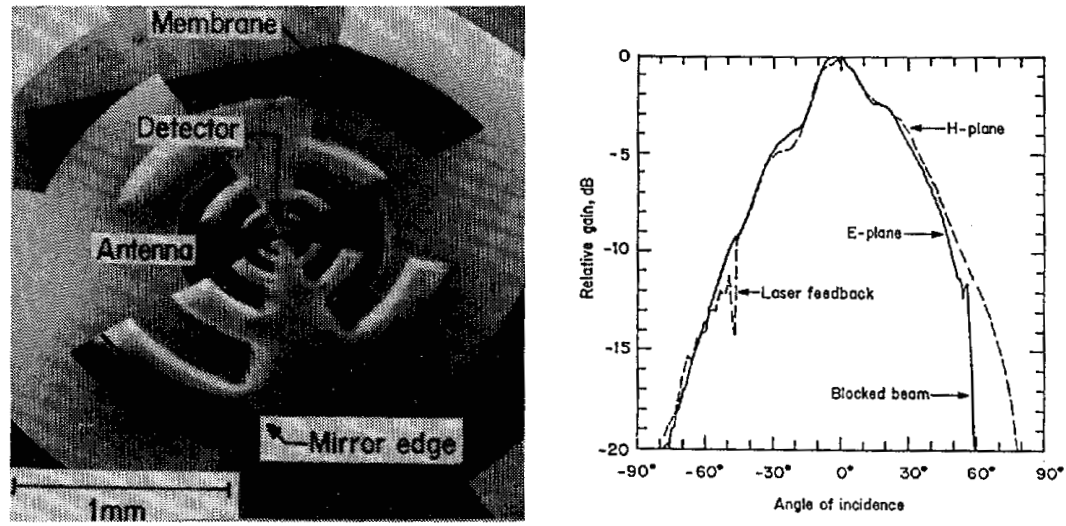

Figure 2. (left) Log-periodic antenna with back-shorting mirror. (right) Measured $\mathrm{E}$ and $\mathrm{H}$ plane patterns at $700 \mathrm{GHz}$ without mirror, taken from top side of the wafer.
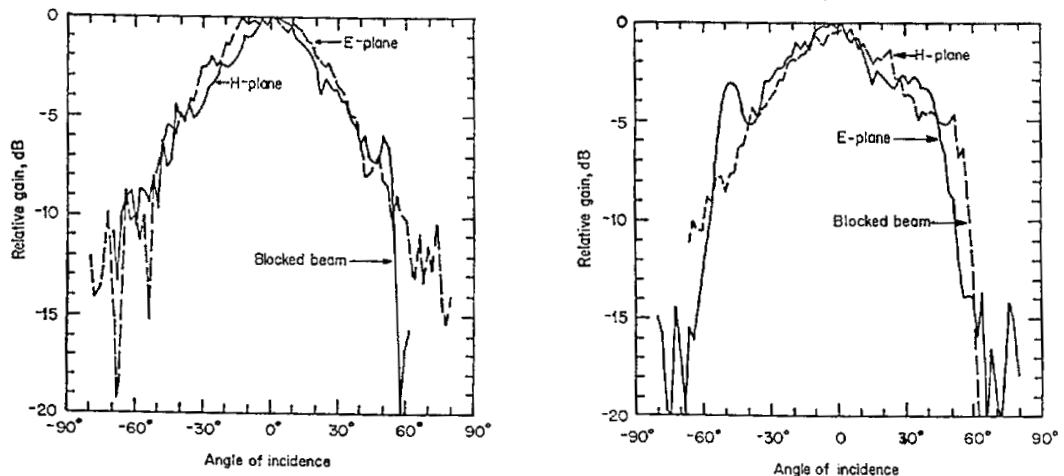

Figure 3. Measured $\mathrm{E}$ and $\mathrm{H}$ plane patterns at $380 \mathrm{GHz}$ (left) and $167 \mathrm{GHz}$ (right) without mirror, taken from top side of the wafer.

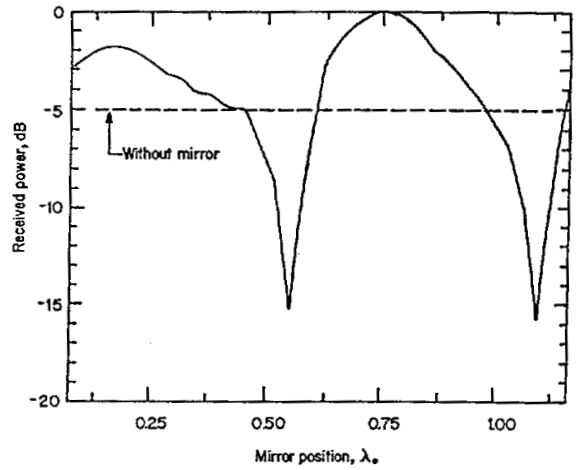

Figure 4. Measured received power vs. mirror position at $380 \mathrm{GHz}$. 


\section{Log-periodic imaging array}

The antennas are single-toothed self-complementary log periodics (Fig. 5) designed for a frequency of $700 \mathrm{GHz}$ with a spacing of $0.9 \lambda$. The polarization axis is not directed along the tooth, but is shifted clockwise $20^{\circ}$ toward the array axis. Since the polarization does not match the array axis, the $E$ field was rotated by a half-wave plate to yield the maximum signal from the detector, and the horizontal-plane (array-axis) and vertical-plane (bow-axis) patterns were measured (Fig. 3).
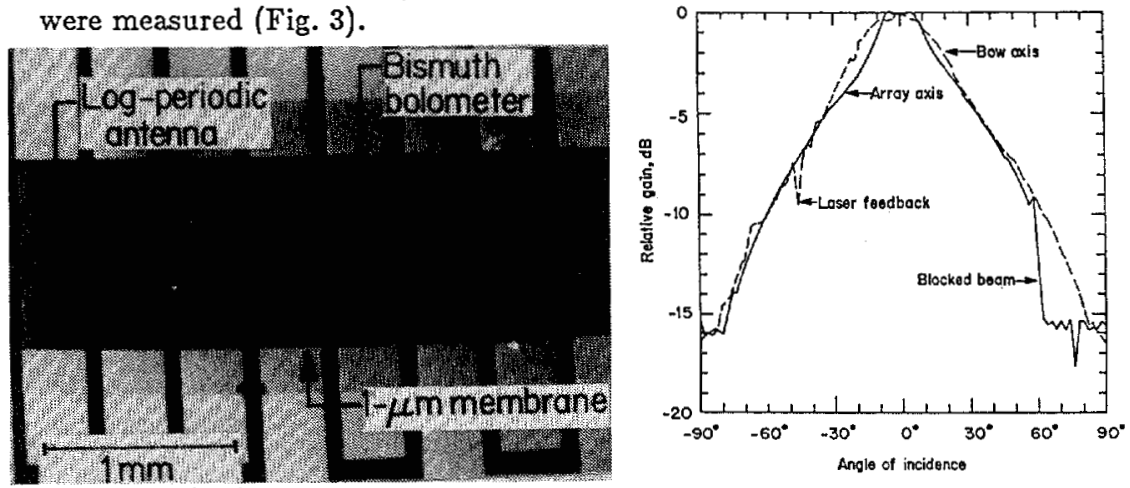

Figure 5. (left) Log-periodic imaging array. (right) Measured array-axis and bow axis patterns on a central element without mirror, taken from top side of the wafer.

\section{Acknowledgements}

We thank Dr. Peter Siegel at the National Radio Astronomy Observatory for suggesting the use of log-periodic antennas. We thank Dr. W. R. Snow at Pacific Western Systems for his advice in the deposition of the silicon-oxynitride layer. This work was supported by contracts from the Army Research Office, the Department of Energy and JPL's program in innovative research.

\section{References}

[1] D. B. Rutledge, D. P. Neikirk and D. P. Kasilingam, "Integrated circuit antennas," in Infrared and Millimeter Waves, vol.10, K.J.Button, ed., Academic Press, New York, pp.1-90, 1983.

[2] C. E. Zah, D. P. Kasilingam, J. S. Smith and D. B. Rutledge, "Millimeter wave monolithic Schottky diode imaging array," Int. J. of Infrared and Millimeter Waves 6, pp. 981-987, 1985.

[3] K. E. Peterson, "Silicon as a mechanical material," Proc. of the IEEE 70, pp. 420-457, 1982.

[4] D. P. Neikirk, W. W. Lam and D. B. Rutledge, "Far-infrared microbolometer detectors," Int. J. of Infrared and Millimeter Waves 5, pp. 245-278, 1984.

[5] R. H. DuHamel and D. E. Isbell, "Broadband logarithmically periodic antenna structure," IRE National Convention Record, Part I, pp. 119-128, 1957.

[6] P. H. Siegel, "A planar log-periodic mixtenna for millimeter and submillimeter wavelengths," IEEE Int. Microwave Symposium, pp.649-652, 1986. 